JINR preprint E2-94-39

hep-th/9402067

\title{
COADDITIVE DIFFERENTIAL COMPLEXES ON QUANTUM GROUPS AND QUANTUM SPACES
}

\author{
A.A.VLADIMIROV*॰ \\ Bogolubov Laboratory of Theoretical Physics, \\ Joint Institute for Nuclear Research, \\ Dubna, Moscow region 141980, Russia
}

\begin{abstract}
A regular way to define an additive coproduct (or coaddition) on the $q$-deformed differential complexes is proposed for quantum groups and quantum spaces related to the Hecke-type $R$-matrices. Several examples of braided coadditive differential bialgebras (Hopf algebras) are presented.
\end{abstract}

* E-mail: alvladim@thsun1.jinr.dubna.su

$\diamond$ Work supported in part by the Russian Foundation of Fundamental Research (grant No. 93-02-3827). 
1. Recently, an additive version of coproduct (or rather coaddition) has been observed in various quantum (q-deformed) algebras [1, 2, 3]. While in the ordinary Lie algebras this additional algebraic structure is quite natural and almost trivial, in a $q$-deformed situation it requires nontrivial braiding rules [1], thus making the corresponding quantum algebras the braided coadditive bialgebras (actually, Hopf algebras).

A related and very interesting question is a possible bialgebra structure of differential complexes, i.e., a concept of differential bialgebras [5, 6]. Brzezinski [7] has shown that the existence of a bialgebra of this type means the bicovariance of the corresponding differential calculus [8, 9, 10].

Therefore, ones interest in the braided coaddition in differential complexes could be at least threefold:

- it is interesting by itself, as an additional algebraic structure;

- it can provide us with a purely Hopf-algebraic criterion for selecting $q$-deformed differential calculi;

- it might play a role of a "shift" in the physical interpretation of the corresponding quantum space.

In [11], among other examples, several coadditive differential bialgebras have been obtained. The aim of the present paper is to give a systematic approach to this problem for quantum algebras generated by the $R$-matrices of the Hecke type (for instance, the $G L_{q}(N)$ ones [12]). Proceeding in this way, we recover the results of [11], describe a regular (and very simple) method to prove consistency (associativity) of the relevant braiding relations, and find a braided coadditive differential Hopf-algebra structure on the corresponding quantum group.

This paper has developed from my attempts to interpret eqs.(47),(48) (see below) found by A.Isaev [11]. I appreciate this contribution of his to the present work.

2. Principal ideas of this paper can be best explained by using the well accustomed quantum hyperplane

$$
R_{12} x_{1} x_{2}=q x_{2} x_{1}
$$

as an example. We adopt the following notation [13, 11]:

$$
P_{12} R_{12} \equiv \hat{R}_{12} \equiv R, \quad \hat{R}_{23} \equiv R^{\prime}, \quad R^{-1} \equiv \bar{R}, \quad q^{-1} \equiv \bar{q}
$$

and also, for any $a$,

$$
a_{1} \equiv a, \quad a_{2} \equiv a^{\prime}, \quad a_{3} \equiv a^{\prime \prime}, \quad a \otimes 1 \equiv a, \quad 1 \otimes a \equiv \tilde{a} .
$$

For instance, the Yang-Baxter equation and the Hecke condition for the $R$-matrix look now, respectively,

$$
R R^{\prime} R=R^{\prime} R R^{\prime}
$$

and

$$
R-\bar{R}=q-\bar{q} \equiv \lambda \quad \text { or } \quad R^{2}=1+\lambda R .
$$

Our aim is to suppress explicit numerical indices (numbers of the corresponding auxiliary spaces) in formulae like (1) in order not to mix them with others that we shall need very soon. 

by

Really, the whole differential complex [14] on the quantum hyperplane (11) is defined

$$
\left\{\begin{array}{l}
R x x^{\prime}=q x x^{\prime}, \\
R d x x^{\prime}=\bar{q} x d x^{\prime}, \\
R d x d x^{\prime}=-\bar{q} d x d x^{\prime} .
\end{array}\right.
$$

Adding formally to this set of equations an extra one,

$$
d x x^{\prime}=q \bar{R} x d x^{\prime}-\lambda q d x x^{\prime}
$$

which trivially follows from the second line in (6), one can recast $(6),(17)$ into the matrix form

$$
\chi_{2} \chi_{1}^{\prime}=Y_{12} \chi_{1} \chi_{2}^{\prime}
$$

where

$$
\chi=\left(\begin{array}{c}
x \\
d x
\end{array}\right), \quad Y_{12}=q\left(\begin{array}{cccc}
\bar{R} & \cdot & \cdot & \cdot \\
\cdot & \bar{R} & -\lambda & \cdot \\
\cdot & \cdot & R & \cdot \\
\cdot & \cdot & \cdot & -R
\end{array}\right)
$$

dots are zeros, and the meaning of numerical indices in (8) is, of course, not the same as in (11). It should be noted that the explicit form (9) chosen here for $Y_{12}$ is by no means unique.

Now we are to employ the matrix representation (8) for demonstrating that the differential complex (6) admits coaddition of the form

$$
\Delta(x)=x \otimes 1+1 \otimes x \equiv x+\tilde{x}, \quad \Delta(d x)=d x+d \tilde{x}
$$

or, in short notation,

$$
\Delta(\chi)=\chi+\tilde{\chi}
$$

From earlier papers on the subject [1], 11], we learn that this can be only possible when a nontrivial braiding map $\Psi: \tilde{\Omega} \otimes \Omega \rightarrow \Omega \otimes \tilde{\Omega}$ is used to commute elements with and without a tilde from two independent copies of our differential complex $\Omega$. Explicitly,

$$
(1 \otimes a)(b \otimes 1) \equiv \tilde{a} b=\Psi(a \otimes b)
$$

In the case (8), a natural Ansatz for the braiding is

$$
\tilde{\chi}_{2} \chi_{1}^{\prime}=Z_{12} \chi_{1} \tilde{\chi}_{2}^{\prime}
$$

where $Z$ is a $4 \times 4$-matrix whose elements may themselves depend on $R$.

The first restriction on $Z$ is caused by the graded nature of the differential complex (6). This leads to

$$
Z_{12}=\left(\begin{array}{cccc}
\alpha & \cdot & \cdot & \cdot \\
\cdot & \gamma & \delta & \cdot \\
\cdot & \mu & \beta & \cdot \\
\cdot & \cdot & \cdot & \nu
\end{array}\right)
$$


Further, the result of external differentiation of (13) must be consistent with (13) itself. Taking into account $d^{2}=0$ and the graded Leibnitz rule, we come to

$$
\alpha=\beta+\delta, \quad \gamma=\delta-\nu, \quad \mu=\beta+\nu
$$

The next step is to ensure the key property of $\Delta$, i.e.

$$
\Delta\left(\chi_{2}\right) \Delta\left(\chi_{1}^{\prime}\right)=Y_{12} \Delta\left(\chi_{1}\right) \Delta\left(\chi_{2}^{\prime}\right)
$$

This boils down to verification of

$$
\tilde{\chi}_{2} \chi_{1}^{\prime}+\chi_{2} \tilde{\chi}_{1}^{\prime}=Y_{12} \tilde{\chi}_{1} \chi_{2}^{\prime}+Y_{12} \chi_{1} \tilde{\chi}_{2}^{\prime},
$$

which, with the help of (13), transforms to

$$
\left[Y_{12} Z_{21}+\left(Y_{12}-Z_{12}\right) P_{12}-\mathbf{1}\right] \chi_{2} \tilde{\chi}_{1}^{\prime}=0 \text {. }
$$

We have to put the expression in square brackets to zero. This results in the following new constraints:

$$
\beta=(\delta+1) q R, \quad(\nu+1)(R+\bar{q})=0 .
$$

At last, we must guarantee that our braiding (13) obeys so-called hexagon identities [15] or, equivalently, that our commutation rules for elements with and without a tilde are associative. To do this, we perform a reordering

$$
\tilde{\chi}_{3} \chi_{2}^{\prime} \chi_{1}^{\prime \prime} \rightarrow \chi_{1} \chi_{2}^{\prime} \tilde{\chi}_{3}^{\prime \prime}
$$

in two different ways, using (8), (13) and

$$
\chi_{2}^{\prime} \chi_{1}^{\prime \prime}=Y_{12}^{\prime} \chi_{1}^{\prime} \chi_{2}^{\prime \prime}, \quad \tilde{\chi}_{2}^{\prime} \chi_{1}^{\prime \prime}=Z_{12}^{\prime} \chi_{1}^{\prime} \tilde{\chi}_{2}^{\prime \prime}
$$

where $Y^{\prime}$ and $Z^{\prime}$ mean that a substitution $R \rightarrow R^{\prime}$ in the corresponding elements of $Y$ and $Z$ has to be carried out. Following this strategy, we finally obtain

$$
Y_{12}^{\prime} Z_{13} Z_{23}^{\prime}=Z_{23} Z_{13}^{\prime} Y_{12}
$$

(A similar relation for $Y$,

$$
Y_{12}^{\prime} Y_{13} Y_{23}^{\prime}=Y_{23} Y_{13}^{\prime} Y_{12}
$$

which expresses the associativity of the original algebra (6), is of course readily verified).

Rewriting the matrix relations (22) in the component form, we immediately encounter

$$
R^{\prime}(\beta+\nu) \delta^{\prime}=\delta\left(\beta^{\prime}+\nu^{\prime}\right) \bar{R}=0 .
$$

The only way out is to nullify $\delta$ or $\beta+\nu$. Let us first consider the latter possibility. Then, due to (19),

$$
\nu=-\beta, \quad(\beta-1)(R+\bar{q})=0, \quad \beta+\delta=\bar{q} \bar{R},
$$


and the matrix $Z_{12}$ becomes

$$
Z_{12}=\left(\begin{array}{cccc}
\bar{q} \bar{R} & \cdot & \cdot & \cdot \\
\cdot & \bar{q} \bar{R} & \bar{q} \bar{R}-\beta & \cdot \\
\cdot & \cdot & \beta & \cdot \\
\cdot & \cdot & \cdot & -\beta
\end{array}\right)
$$

The remaining relations hidden in (22) yield

$$
\beta \bar{R}^{\prime} \bar{R}=\bar{R}^{\prime} \bar{R} \beta^{\prime}, \quad \beta \beta^{\prime} R=R^{\prime} \beta \beta^{\prime} .
$$

The first of these is identically true whereas the second, together with (25), produces two solutions for $\beta$,

$$
\beta=\bar{q} R \quad \text { or } \beta=q \bar{R},
$$

and, consequently, two possibilities for $Z$,

$$
Z_{12}^{(1)}=\bar{q}\left(\begin{array}{cccc}
\bar{R} & \cdot & \cdot & \cdot \\
\cdot & \bar{R} & -\lambda & \cdot \\
\cdot & \cdot & R & \cdot \\
\cdot & \cdot & \cdot & -R
\end{array}\right), \quad Z_{12}^{(2)}=\bar{R}\left(\begin{array}{cccc}
\bar{q} & \cdot & \cdot & \cdot \\
\cdot & \bar{q} & -\lambda & \cdot \\
\cdot & \cdot & q & \cdot \\
\cdot & \cdot & \cdot & -q
\end{array}\right)
$$

In the explicit form this reads:

$$
\begin{aligned}
& \left\{\begin{array}{l}
\tilde{x} x^{\prime}=\bar{q} \bar{R} x \tilde{x}^{\prime}, \\
d \tilde{x} x^{\prime}=\bar{q} \bar{R} x d \tilde{x}^{\prime}-\lambda \bar{q} d x \tilde{x}^{\prime}, \\
\tilde{x} d x^{\prime}=\bar{q} R d x \tilde{x}^{\prime} \\
d \tilde{x} d x^{\prime}=-\bar{q} R d x d \tilde{x}^{\prime} ;
\end{array}\right. \\
& \left\{\begin{array}{l}
\tilde{x} x^{\prime}=\bar{q} \bar{R} x \tilde{x}^{\prime} \\
d \tilde{x} x^{\prime}=\bar{q} \bar{R} x d \tilde{x}^{\prime}-\lambda \bar{R} d x \tilde{x}^{\prime}, \\
\tilde{x} d x^{\prime}=q \bar{R} d x \tilde{x}^{\prime} \\
d \tilde{x} d x^{\prime}=-q \bar{R} d x d \tilde{x}^{\prime} .
\end{array}\right.
\end{aligned}
$$

The other solution of (24), $\delta=0$, produces matrices $\bar{Z}_{21}^{(1)}$ and $\bar{Z}_{21}^{(2)}$ instead of (29). This evidently corresponds to changing the position of a tilde $(\tilde{\chi} \leftrightarrow \chi, \tilde{x} \leftrightarrow x)$ in (13), (30) and (31), i.e., to the inverse braiding transformation $\Psi^{-1}$. We thus recover the results of [1] and, moreover, prove that they exhaust all the allowed braiding relations within the homogeneous Ansatz (13). It should be also stressed that the representations like (8) and (13) are extremely convenient for proving associativity (resp. consistency) of appropriate multiplication or braiding relations.

3. Now we proceed to the case of the braided matrix algebra $B M_{q}(N)$ [16, 17] with the generators $\left\{1, u_{j}^{i}\right\}$, forming the $N \times N$-matrix $u$, and relations

$$
R_{21} u_{2} R_{12} u_{1}=u_{1} R_{21} u_{2} R_{12} \text {. }
$$


The corresponding differential complex is described in [18, 19]. In our conventions (note $u_{1} \equiv u$ ) it reads

$$
\left\{\begin{array}{l}
R u R u=u R u R, \\
R u R d u=d u R u \bar{R}, \\
R d u R d u=-d u R d u \bar{R}
\end{array}\right.
$$

(unlike (6), there are no primes in these equations). The appropriate coaddition is also known (see [2] for the $B M_{q}(N)$ itself and [1] for (33) as a whole). Here we wish to reproduce the results of [11] through the matrix formalism developed in the previous section.

Let us rewrite(33) in the form

$$
\varphi_{2} R \varphi_{1}=V_{12} \varphi_{1} R \varphi_{2} R,
$$

where

$$
\varphi=\left(\begin{array}{c}
u \\
d u
\end{array}\right), \quad V_{12}=\left(\begin{array}{cccc}
\bar{R} & \cdot & \cdot & \cdot \\
\cdot & R & \cdot & \cdot \\
\cdot & -\lambda & \bar{R} & \cdot \\
\cdot & \cdot & \cdot & -R
\end{array}\right),
$$

and try to introduce the braiding relations

$$
\tilde{\varphi}_{2} R \varphi_{1}=W_{12} \varphi_{1} R \tilde{\varphi_{2}} R
$$

which make

$$
\Delta(\varphi)=\varphi+\tilde{\varphi}
$$

a consistent coproduct. From (34) and (36) we deduce

$$
W_{12} \varphi_{1} R \tilde{\varphi}_{2} R+\varphi_{2} R \tilde{\varphi}_{1}=V_{12} W_{21} \varphi_{2} R \tilde{\varphi}_{1} R^{2}+V_{12} \varphi_{1} R \tilde{\varphi}_{2} R .
$$

With the help of the Hecke condition (5) we get

$$
\left(V_{12} W_{21}-1\right) \varphi_{2} R \tilde{\varphi}_{1}+\left[\lambda V_{12} W_{21}+\left(V_{12}-W_{12}\right) P_{12}\right] \varphi_{2} R \tilde{\varphi}_{1} R=0 .
$$

A solution is

$$
W_{12}=\bar{V}_{21}=\left(\begin{array}{cccc}
R & \cdot & \cdot & \cdot \\
\cdot & R & \lambda & \cdot \\
\cdot & \cdot & \bar{R} & \cdot \\
\cdot & \cdot & \cdot & -\bar{R}
\end{array}\right) .
$$

Another possible braiding is

$$
\tilde{\varphi}_{2} R \varphi_{1}=V_{12} \varphi_{1} R \tilde{\varphi_{2}} \bar{R},
$$

inspired by the following equivalent version of (34):

$$
\varphi_{2} R \varphi_{1}=\bar{V}_{21} \varphi_{1} R \varphi_{2} \bar{R}
$$

Of course, this corresponds to the inverse braiding map with respect to (36), (40). 
Another pair of mutually inverse solutions can be obtained if one represents (33) as

$$
\eta_{2} R \eta_{1}=R \eta_{1} R \eta_{2} V_{12}^{T}=\bar{R} \eta_{1} R \eta_{2} \bar{V}_{21}^{T}
$$

where $\eta$ is now a row instead of a column:

$$
\eta=(u, d u), \quad V_{12}^{T}=\left(\begin{array}{cccc}
\bar{R} & \cdot & \cdot & \cdot \\
\cdot & R & -\lambda & \cdot \\
\cdot & \cdot & \bar{R} & \cdot \\
\cdot & \cdot & \cdot & -R
\end{array}\right) .
$$

In this case, both

$$
\tilde{\eta}_{2} R \eta_{1}=R \eta_{1} R \tilde{\eta}_{2} \bar{V}_{21}^{T}
$$

and

$$
\tilde{\eta}_{2} R \eta_{1}=\bar{R} \eta_{1} R \tilde{\eta}_{2} V_{12}^{T}
$$

are consistent braiding relations. Associativity of (36),(41),(45) and (46) (i.e. the identities like $\left.W_{12} W_{13}^{\prime} V_{23}=V_{23}^{\prime} W_{13} W_{12}^{\prime}\right)$ and their compatibility with the Leibnitz rule are easily confirmed.

In the component form, (36) and (45) look, respectively, as

$$
\begin{aligned}
& \left\{\begin{array}{l}
\tilde{u} R u=R u R \tilde{u} R, \\
d \tilde{u} R u=R u R d \tilde{u} R+\lambda d u R \tilde{u} R, \\
\tilde{u} R d u=\bar{R} d u R \tilde{u} R, \\
d \tilde{u} R d u=-\bar{R} d u R d \tilde{u} R ;
\end{array}\right. \\
& \left\{\begin{array}{l}
\tilde{u} R u=R u R \tilde{u} R, \\
d \tilde{u} R u=R u R d \tilde{u} R+\lambda R d u R \tilde{u}, \\
\tilde{u} R d u=R d u R \tilde{u} \bar{R}, \\
d \tilde{u} R d u=-R d u R d \tilde{u} \bar{R} ;
\end{array}\right.
\end{aligned}
$$

eqs. (41) and (46) being obtained from these via $u \leftrightarrow \tilde{u}$. We recover the corresponding results given in [11].

4. Consider at last the familiar matrix quantum group

$$
R_{12} T_{1} T_{2}=T_{2} T_{1} R_{12},
$$

which also has a braided coaddition [3]. Its differential complex is known too [20. In the notation (2),(3) it looks like

$$
\left\{\begin{array}{l}
R T T^{\prime}=T T^{\prime} R \\
R d T T^{\prime}=T d T^{\prime} \bar{R} \\
R d T d T^{\prime}=-d T d T^{\prime} \bar{R}
\end{array}\right.
$$

Let us show that the algebra $(\sqrt[50]{ })$ as a whole admits a coaddition

$$
\Delta(\theta)=\theta+\tilde{\theta}, \quad \theta \equiv\left(\begin{array}{c}
T \\
d T
\end{array}\right)
$$


Really, eq.(50) is easily rewritten as

$$
\theta_{2} \theta_{1}^{\prime}=N_{12} \theta_{1} \theta_{2}^{\prime} R, \quad N_{12}=\left(\begin{array}{cccc}
\bar{R} & \cdot & \cdot & \cdot \\
\cdot & \bar{R} & -\lambda & \cdot \\
\cdot & \cdot & R & \cdot \\
\cdot & \cdot & \cdot & -R
\end{array}\right) .
$$

In complete analogy with the preceding section, one finds that the mutually inverse braiding relations

$$
\begin{gathered}
\tilde{\theta}_{2} \theta_{1}^{\prime}=\bar{N}_{21} \theta_{1} \tilde{\theta}_{2}^{\prime} R, \\
\tilde{\theta}_{2} \theta_{1}^{\prime}=N_{12} \theta_{1} \tilde{\theta}_{2}^{\prime} \bar{R}
\end{gathered}
$$

satisfy all the requirements. If, otherwise, eq.(50) is recast into the form

$$
\xi_{2} \xi_{1}^{\prime}=R \xi_{1} \xi_{2}^{\prime} N_{12}^{T}
$$

with $\xi$ being a row, $\xi=(T, d T)$, then the following pair of mutually inverse braidings is produced:

$$
\begin{aligned}
& \tilde{\xi}_{2} \xi_{1}^{\prime}=R \xi_{1} \tilde{\xi}_{2}^{\prime} \bar{N}_{21}^{T}, \\
& \tilde{\xi}_{2} \xi_{1}^{\prime}=\bar{R} \xi_{1} \tilde{\xi}_{2}^{\prime} N_{12}^{T} .
\end{aligned}
$$

In the component form:

$$
\begin{aligned}
& \left\{\begin{array}{l}
\tilde{T} T^{\prime}=R T \tilde{T}^{\prime} R \\
d \tilde{T} T^{\prime}=\bar{R} T d \tilde{T}^{\prime} R \\
\tilde{T} d T^{\prime}=R d T \tilde{T}^{\prime} R+\lambda T d \tilde{T}^{\prime} R, \\
d \tilde{T} d T^{\prime}=-\bar{R} d T d \tilde{T}^{\prime} R
\end{array}\right. \\
& \left\{\begin{array}{l}
\tilde{T} T^{\prime}=R T \tilde{T}^{\prime} R \\
d \tilde{T} T^{\prime}=R T d \tilde{T}^{\prime} \bar{R} \\
\tilde{T} d T^{\prime}=R d T \tilde{T}^{\prime} R+\lambda R T d \tilde{T}^{\prime}, \\
d \tilde{T} d T^{\prime}=-R d T d \tilde{T}^{\prime} \bar{R}
\end{array}\right.
\end{aligned}
$$

two other sets are obtained from these by $\tilde{T} \leftrightarrow T$.

All the above examples lead us to the conclusion that the braided coaddition appears to be a quite natural algebraic structure for the differential complexes on the quadratic quantum algebras generated by the Hecke-type $R$-matrices. The corresponding (braided) counit obeys $\varepsilon(1)=1$ and equals zero on other generators. Moreover, a braided antipode is easily introduced:

$$
S(1)=1, \quad S(a)=-a, \quad S(d a)=-d a \quad(a=x, u, T) .
$$

Consequently, all the braided coadditive differential bialgebras considered in this paper are, in fact, braided Hopf algebras. 


\section{References}

[1] S.Majid, J.Math.Phys. 34 (1993) 2045.

[2] U.Meyer, Preprint DAMTP/93-45, 1993.

[3] S.Majid, Preprint DAMTP/93-57, 1993.

[4] S.Majid, Beyond supersymmetry and quantum symmetry (an introduction to braided groups and braided matrices), in: M.-L. Ge and H.J. de Vega, editors, Quantum Groups, Integrable Statistical Models and Knot Theory, pp. 231-282, World.Sci., 1993.

[5] G.Maltsiniotis, C.R.Acad.Sci.Paris 331 (1990) 831; Comm.Math.Phys. 151 (1993) 275 .

[6] Yu.I.Manin, Teor.Mat.Fiz. 92 (1992) 425.

[7] T.Brzezinski, Lett.Math.Phys. 27 (1993) 287.

[8] S.L.Woronowicz, Comm.Math.Phys. 122 (1989) 125.

[9] B.Jurčo, Lett.Math.Phys. 22 (1991) 177.

[10] P.Aschieri and L.Castellani, Int.J.Mod.Phys. A8 (1993) 1667.

[11] A.P.Isaev and A.A.Vladimirov, JINR preprint E2-94-32, Dubna, 1994 (hep-th/9402024).

[12] L.D.Faddeev, N.Yu.Reshetikhin and L.A Takhtajan, Algebra i Analiz 1 Vol.1 (1989) 178; English transl: Leningr.Math.J. 1 (1990) 193.

[13] A.P.Isaev and P.N.Pyatov, JINR preprint E2-93-416, Dubna, 1993 (hep-th/9311112).

[14] J.Wess and B.Zumino, Nucl.Phys. B (Proc.Suppl.) 18B (1990) 302.

[15] S.Majid, Int.J.Mod.Phys. A 5 (1990) 1.

[16] P.P.Kulish and E.K.Sklyanin, J.Phys. A 25 (1992) 5963; P.P.Kulish and R.Sasaki, Progr.Theor.Phys. 89 (1993) 741.

[17] S.Majid, J.Math.Phys. 32 (1991) 3246; ibid. 34 (1993) 1176.

[18] O.Ogievetsky, W.B.Schmidke, J.Wess and B.Zumino, Comm.Math.Phys. 150 (1992) 495.

[19] J.A. de Azcarraga, P.P.Kulish and F.Rodenas, Valencia University preprint FTUV 93-36, 1993; P.P.Kulish, Valencia University preprint FTUV 93-54, 1993.

[20] A.Sudbery, Phys.Lett. 284B (1992) 61. 